\title{
Neuroprotective and Axon Growth-Promoting Effects following Inflammatory Stimulation on Mature Retinal Ganglion Cells in Mice Depend on Ciliary Neurotrophic Factor and Leukemia Inhibitory Factor
}

\author{
Marco Leibinger, ${ }^{1 \star}$ Adrienne Müller, ${ }^{1 \star}$ Anastasia Andreadaki, ${ }^{1}$ Thomas G. Hauk, ${ }^{1}$ Matthias Kirsch, ${ }^{2}$ \\ and Dietmar Fischer ${ }^{1}$ \\ ${ }^{1}$ Department of Experimental Neurology, University of Ulm, 89081 Ulm, Germany, and ²Department of Anatomy, University of Freiburg, 79098 Freiburg, \\ Germany
}

\begin{abstract}
After optic nerve injury retinal ganglion cells (RGCs) normally fail to regenerate axons in the optic nerve and undergo apoptosis. However, lens injury (LI) or intravitreal application of zymosan switch RGCs into an active regenerative state, enabling these neurons to survive axotomy and to regenerate axons into the injured optic nerve. Several factors have been proposed to mediate the beneficial effects of LI. Here, we investigated the contribution of glial-derived ciliary neurotrophic factor (CNTF) to LI-mediated regeneration and neuroprotection using wild-type and CNTF-deficient mice. In wild-type mice, CNTF expression was strongly upregulated in retinal astrocytes, the JAK/STAT3 pathway was activated in RGCs, and RGCs were transformed into an active regenerative state after LI. Interestingly, retinal LIF expression was correlated with CNTF expression after LI. In CNTF-deficient mice, the neuroprotective and axon growth-promoting effects of LI were significantly reduced compared with wild-type animals, despite an observed compensatory upregulation of LIF expression in CNTF-deficient mice. The positive effects of LI and also zymosan were completely abolished in CNTF/LIF double knock-out mice, whereas LI-induced glial and macrophage activation was not compromised. In culture CNTF and LIF markedly stimulated neurite outgrowth of mature RGCs. These data confirm a key role for CNTF in directly mediating the neuroprotective and axon regenerative effects of inflammatory stimulation in the eye and identify LIF as an additional contributing factor.
\end{abstract}

\section{Introduction}

Retinal ganglion cells (RGCs) of mature mammals normally fail to regenerate axons into the damaged optic nerve, and instead, undergo apoptotic cell death (Berkelaar et al., 1994; Berry et al., 1996, 1999; Fischer et al., 2000). However, inflammatory stimulation in the eye, by intravitreal application of $\beta / \gamma$-crystallins, releasing $\beta / \gamma$-crystallins from the injured lens, by intravitreal injection of zymosan or the toll-like receptor 2 agonist $\mathrm{Pam}_{3} \mathrm{Cys}$, delays axotomy-induced death and transforms RGCs into an active regenerative state (Fischer et al., 2000, 2004b, 2008; Yin et al., 2003; Hauk et al., 2009). In this regenerative state, which is associated with a dramatic change in gene expression, RGCs can survive axotomy and regenerate axons over several millimeters beyond the lesion site into the optic nerve (Fischer et al., 2000, 2001, 2004b; Leon et al., 2000; Lorber et al., 2002, 2005a; Yin et al., 2003; Pernet and Di Polo, 2006). Similarly, lens injury (LI) in the

Received June 8, 2009; revised 0ct. 7, 2009; accepted 0ct. 10, 2009.

This work was supported by the State of Baden-Württemberg and the German Research Foundation (DFG). We thank Prof. M. Sendtner, University of Würzburg, Würzburg, Germany, for providing us with CNTF-deficient mice. We thank Vetrivel Sengottuvel and Mareike Caesar for critical comments on this manuscript.

${ }^{*} M$.L. and A.M. contributed equally to this work.

Correspondence should be addressed to Prof. Dr. Dietmar Fischer, Department of Experimental Neurology, University of Ulm, Albert-Einstein-Allee 11, 89081 Ulm, Germany. E-mail: dietmar.fischer@uni-ulm.de.

DOI:10.1523/JNEUROSCI.2770-09.2009

Copyright $\odot 2009$ Society for Neuroscience $\quad 0270-6474 / 09 / 2914334-08 \$ 15.00 / 0$ adult mouse also stimulates RGC axon regeneration in vivo and promotes enhanced RGC neurite outgrowth in vitro (Lorber et al., 2005a,b). Intravitreal application of $\beta / \gamma$-crystallins, LI, intravitreal injection of zymosan or $\mathrm{Pam}_{3} \mathrm{Cys}$ are associated with an activation of retinal astrocytes, Müller cells, and microglia as well as an infiltration of macrophages into the vitreous body (Leon et al., 2000; Lorber et al., 2005a; Pernet and Di Polo, 2006; Hauk et al., 2008, 2009). However, controversy exists about the cells and factors mediating the beneficial effects of inflammatory stimulation in the eye. In addition to retinal glia-derived factors, macrophage-derived oncomodulin has been proposed to be the principal mediator of these effects (Yin et al., 2003, 2006; Müller et al., 2007; Hauk et al., 2008; Lorber et al., 2008). However, macrophage depletion in the eye does not significantly abolish the neuroprotective effects or axon growth-promoting properties of LI (Hauk et al., 2008). Moreover, expression levels of oncomodulin in the inner eye are low and not significantly elevated after macrophage infiltration (Hauk et al., 2008; Park et al., 2009). In contrast to macrophages, a reduction in the number of activated retinal glia significantly compromised the beneficial results of zymosan (Lorber et al., 2009), and the beneficial effects of LI were stronger correlated with astrocyte/Müller cell activation than with the number of activated macrophages (Hauk et al., 2008; Lorber et al., 2008), suggesting that glial-derived factors are 
the main mediators of these regenerative effects. Astrocytederived ciliary neurotrophic factor (CNTF) has been shown being a key mediator for this phenomenon (Müller et al., 2007). However, glial-derived factors other than CNTF may be additional contributors. In this context leukemia inhibitory factor (LIF) is of particular interest, since the LIF receptor components (gp130 and LIFR $\beta$ ) are also part of the ternary CNTF receptor complex (Taupin et al., 1998; Heinrich et al., 2003; Sarup et al., 2004; Holtmann et al., 2005) and therefore LIF may be sufficient to stimulate axon growth of mature RGCs.

The goal of this study was to unequivocally investigate the contribution of CNTF to the neuroprotective and axon growth-promoting effects of LI by examining the outcome of LI in wild-type and CNTF-deficient mice. Our results demonstrate that, despite a compensatory upregulation of LIF, the beneficial effects of inflammatory stimulation were strongly compromised in CNTF-deficient mice and completely abolished in CNTF/LIF double knock-out mice without affecting macrophage activation. These data highlight the role of glialderived CNTF as a key factor mediating the neuroprotective and axon growth-promoting effects of LI and identify LIF as an additional contributing factor.

\section{Materials and Methods}

Optic nerve crush, intravitreal injection, and lens injury. Two to 3-monthold adult wild-type, $\mathrm{CNTF}^{-1-}$, and CNTF/LIF double knock-out mice were used for all experiments. Breeding pairs of $\mathrm{CNTF}^{-/-}$and $\mathrm{LIF}^{+/-}$ animals (Sendtner et al., 1996) were generously provided by Prof. M. Sendtner (University of Würzburg, Germany) and maintained on a C57BL/6 background. CNTF/LIF double knock-out mice were produced by crossbreeding male $\mathrm{CNTF}^{-1-} / \mathrm{LIF}^{-1-}$ and female $\mathrm{CNTF}^{-1-} / \mathrm{LIF}^{+/-}$ animals, which were also obtained from M. Sendtner. Control mice (C57BL/6) were obtained from Charles River and were housed under the same conditions for at least $10 \mathrm{~d}$ before being used in experiments. All mice were maintained on a $12 \mathrm{~h}$ light/dark cycle with ad libitum access to food and water. In addition to genotyping, the knock-out of CNTF and LIF was also confirmed by using immunohistochemistry or Western blot analysis to show the lack of retinal cytokine expression (data not shown). All experimental procedures were approved by the local animal care committee (Regierungspräsidium Tübingen) and were in accordance with the guidelines instituted by German laws for animal experiments. For surgery animals were anesthetized by intraperitoneal injections of ketamine $(60-80 \mathrm{mg} / \mathrm{kg})$ and xylazine $(10-15 \mathrm{mg} / \mathrm{kg})$. The optic nerve was intraorbitally crushed for $10 \mathrm{~s}$ directly behind the eye ball using jewelers' forceps under a dissecting microscope as described previously (Müller et al., 2009). LI was induced by retrolentally puncturing the lens capsule with a glass capillary tip immediately following optic nerve crush. Zymosan $(5 \mu \mathrm{g} / 2 \mu \mathrm{l})$ or the same volume of PBS were intravitreally applied by means of a glass capillary after retrieving the same volume from the anterior chamber. Five days after surgery the retina was removed and protein lysates were prepared ( $n=4$ per group), RNA was extracted ( $n=3$ per group) or cells were isolated for culture experiments ( $n=6-8$ per group). To quantify axonal regeneration into the injured nerves, eyes were isolated with optic nerves attached and prepared for histology $14 \mathrm{~d}$ after surgery ( $n=6-10$ per group).

RNA isolation and quantitative real-time PCR. Total RNA was isolated from retinas using the RNeasy kit (Qiagen) according to the manufacturer's protocol. Quantitation of retinal LIF expression was performed with the QuantiTect SYBR Green RT-PCR Kit (Qiagen), using a LightCycler Instrument (LightCycler System, Roche Diagnostics). The amplification signals were detected in real-time permitting accurate quantification of the amounts of the initial RNA template.

Retinal mRNA (100 ng) was reverse transcribed and the cDNA amplified with specific primers during 50 cycles according to the manufacturer's protocol. All reactions were performed in duplicate and three independent samples (from different eyes) per group were run. Quantitative analysis was performed using the LightCycler software. The specificity of the PCR products from each run was determined and verified with the meltingcurve analysis feature of the LightCycler software. QuantiTect primers, GAPDH_Mm_Gapd_1 SG and Mm_Lif_1_SG QuantiTect were purchased from Qiagen.

Semiquantitative reverse-transcription PCR. Total RNA (100 ng) was reverse transcribed. The following primer sequences were used for RT-PCR: oncomodulin, 5'-CGAGCAACTACTAGATCCTTG-3' (forward) and 5'-CACGTTGCAGAAGAGCTTCA-3' (reverse); GAPDH, 5'-GGCCAAGGTCATCCATGACAACTT-3' (forward) and 5'-CCTGCTTCACCACCTTCTTGATGT-3' (reverse); and CD68, 5' -CGTTACTCTCCTGCCATCCTT-3' (forward) and 5' -CTTAGAGAGAGCAGGTCAAG-3' (reverse). To compare samples in the linear range of amplification, RTPCR was stopped and loaded on agarose gels after 25, 30, 35, and 40 cycles.

Immunohistochemistry. Animals were anesthetized and perfused through the heart with cold saline followed by PBS containing $4 \%$ paraformaldehyde. Eyes with attached optic nerve segments were removed from connective tissue, postfixed for several hours, transferred to $30 \%$ sucrose overnight $\left(4^{\circ} \mathrm{C}\right)$, and embedded in Tissue-Tek (Sakura). Frozen sections were longitudinally cut on a cryostat, thaw-mounted onto coated glass slides (Superfrost Plus, Fisher), and stored at $-80^{\circ} \mathrm{C}$ until further use. A monoclonal antibody against GFAP (Santa Cruz Biotechnology) (1:50), polyclonal antibodies against phospho-STAT3 (pSTAT3) (Tyr705) (Cell Signaling Technology) (1:200), CNTF (Serotec) (1:2000 or 1:3000), CD11b (Serotec) (1:500), and a custom-made antibody against GAP43 (Invitrogen) (1:1000) were used. To detect LIF we used a polyclonal antibody (Santa Cruz Biotechnology) at a concentration of 1:200 and another polyclonal anti-LIF antibody (R\&D Systems) (1:100). The specificity of the LIF and CNTF antibodies were confirmed by the presence of LIF and CNTF staining in the retina of wild-type mice after ONC $+\mathrm{LI}$ and their absence in retinas from CNTF/LIF double knock-out animals. Secondary antibodies included anti-mouse IgG, anti-goat IgG, and anti-rabbit IgG antibodies conjugated to Alexa Fluor 488 or Alexa Fluor 594 (Invitrogen) (1:1000). To stain nuclei, sections were incubated in a solution containing 4',6-diamidino-2-phenylindol (DAPI) for 1 min. Fluorescent sections were covered using Mowiol and analyzed under a fluorescent microscope (Zeiss).

Western blot assays. For retinal lysate preparation mice were killed, and their eyeballs were enucleated and dissected. Isolated retinas were collected in lysis buffer (containing, in mM: 20 Tris HCl, pH 7.5, $10 \mathrm{KCl}, 250$ sucrose, $10 \mathrm{NaF}, 1 \mathrm{DTT}, 0.1 \mathrm{Na}_{3} \mathrm{VO}_{4}, 1 \%$ Triton X-100, 0.1\% SDS) with 1:100 protease inhibitor (Calbiochem). Retinas were homogenized and centrifuged at $2700 \times g$ for $10 \mathrm{~min}$ at $4^{\circ} \mathrm{C}$. The supernatants were analyzed by Western blot assay. Separation of proteins was performed using a $10 \%$ SDS polyacrylamide gel, according to standard protocols (BioRad). After SDS-PAGE, proteins were transferred to nitrocellulose membranes (GE Healthcare). The blots were blocked either in 5\% dried milk or in 2\% ECL Advance blocking agent in Tris-buffered saline-Tween 20 (TBS-T). They were then processed for immunostaining with either an antiserum against rat phospho-STAT3 (Tyr705) (Cell Signaling Technology) (1:5000), a monoclonal antibody against rat $\beta$-actin (Sigma) (1:7500), a monoclonal antibody against GFAP (Santa Cruz Biotechnology) (1:5000), a $\beta$ III-tubulin antibody (TUJ-1) (Babco) (1:1000) and an antibody against LIF (Santa Cruz Biotechnology) (1:6000), a polyclonal antibody against rat CNTF (Serotec) (1:5000), or a custom made antibody against GAP43 (Invitrogen) (1:1000) at $4^{\circ} \mathrm{C}$ overnight. Bound antibodies were visualized with anti-rabbit IgG, anti-sheep IgG, or anti-mouse IgG secondary antibodies conjugated with horseradish peroxidase diluted at 1:80,000 (all Sigma). The antigen-antibody complexes were detected by enhanced chemiluminescence (ECL) (GE Healthcare).

Dissociated retinal cell cultures. Tissue culture plates (4-well plates; Nunc) were coated with poly-D-lysine $(0.1 \mathrm{mg} / \mathrm{ml}$, molecular weight $<300,000 \mathrm{Da})$ (Sigma), rinsed with distilled water, and then air-dried. Wells were then coated with laminin $(20 \mu \mathrm{g} / \mathrm{ml})$ (Sigma). To prepare retinal cell cultures, untreated or pretreated animals were killed by an 
overdose of chloral hydrate solution (14\%). Retinas were rapidly dissected from the eyecups and incubated at $37^{\circ} \mathrm{C}$ for $30 \mathrm{~min}$ in a digestion solution containing papain (16.4 $\mathrm{U} / \mathrm{ml}$, Worthington) and L-cysteine $(0.3 \mathrm{~g} / \mathrm{ml}$, Sigma) in DMEM (Invitrogen). Retinas were then rinsed with DMEM and triturated in $2 \mathrm{ml}$ of DMEM. To remove cell fragments or factors released from the cells in the cell suspension, the cell suspension of one retina was immediately adjusted with DMEM to a volume of 50 $\mathrm{ml}$. Cells were then centrifuged at $900 \times g$ for 5 min and the pellet carefully resuspended in 2.5 $\mathrm{ml}$ (per retina) of DMEM containing B27supplement (Invitrogen) (1:50) and penicillin/ streptomycin (Biochrom) (1:50). Dissociated cells were then passed through a cell strainer (40 $\mu \mathrm{m}$, Falcon) and $300 \mu \mathrm{l}$ of cell suspension were added into each well. The investigator was blinded to the arrangement and identity of the cultures to prevent the introduction of bias to the analysis. In experiments where the potency of CNTF or LIF was assessed the cytokines were applied to the medium at $10,30,50,200$ or 400 $\mathrm{ng} / \mathrm{ml}$ and neurite outgrowth was determined after $3 \mathrm{~d}$. The anti-LIF antibody (R\&D Systems) was applied at $5000 \mathrm{ng} / \mathrm{ml}$. In experiments, where the regenerative state of RGCs was determined after LI, cells were fixed with a paraformaldehyde solution (4\%) and methanol (Sigma) after $24 \mathrm{~h}$ in culture and they were then prepared for immunocytochemical staining with a $\beta$ III-tubulinantibody (TUJ-1) (Babco) (1:2000). All RGCs with regenerated neurites were photographed under a fluorescent microscope $(200 \times)$. The neurite length of RGCs was determined using the ImageJ software. Furthermore, the total number of $\beta$ III-tubulin-positive RGCs with an intact nucleus (DAPI) per well was quantified to evaluate the potential neurotoxic or neuroprotective effects following each treatment. Values for axon outgrowth were determined by the sum of the neurite length per well divided by the total number of RGCs per well, resulting in the average neurite length per RGC. Values were then normalized to the control groups as indicated. The data are given as the mean \pm SEM of four replicate wells. The significance of intergroup difference was evaluated using a one-way ANOVA test, followed by corrections for multiple post hoc tests (Bonferroni-Holm, Tukey). Each experiment was repeated at least four times.

Quantitation of axons in the optic nerve and $R G C s$ in retinal cross-sections. Regeneration was quantified as described previously (Leon et al., 2000; Müller et al., 2007). In brief, under $400 \times$ magnification, we counted the number of GAP43-positive axons in eight sections per case extending $>0.25 \mathrm{~mm}$, $>0.5 \mathrm{~mm}$ or $>1 \mathrm{~mm}$ from the site of injury. These values were normalized to the cross-sectional width of the optic nerve $1 \mathrm{~mm}$. For quantification of RGCs/retinal cross-sections, sections through the center of the retina were stained with an antibody $\beta$ III-tubulin (TUJ1) as described above. The numbers of $\beta$ III-tubulin-positive cells per section were counted in $4-6$ sections per case, averaged per case, then averaged across all similarly treated animals to obtain a group means and SE as described previously (Fischer et al., 2004a,b; Müller et al., 2007). The significances of intergroup differences were evaluated using a one-way (ANOVA) test, followed by corrections for multiple post hoc tests (Bonferroni-Holm, Tukey) protein was loaded per lane.
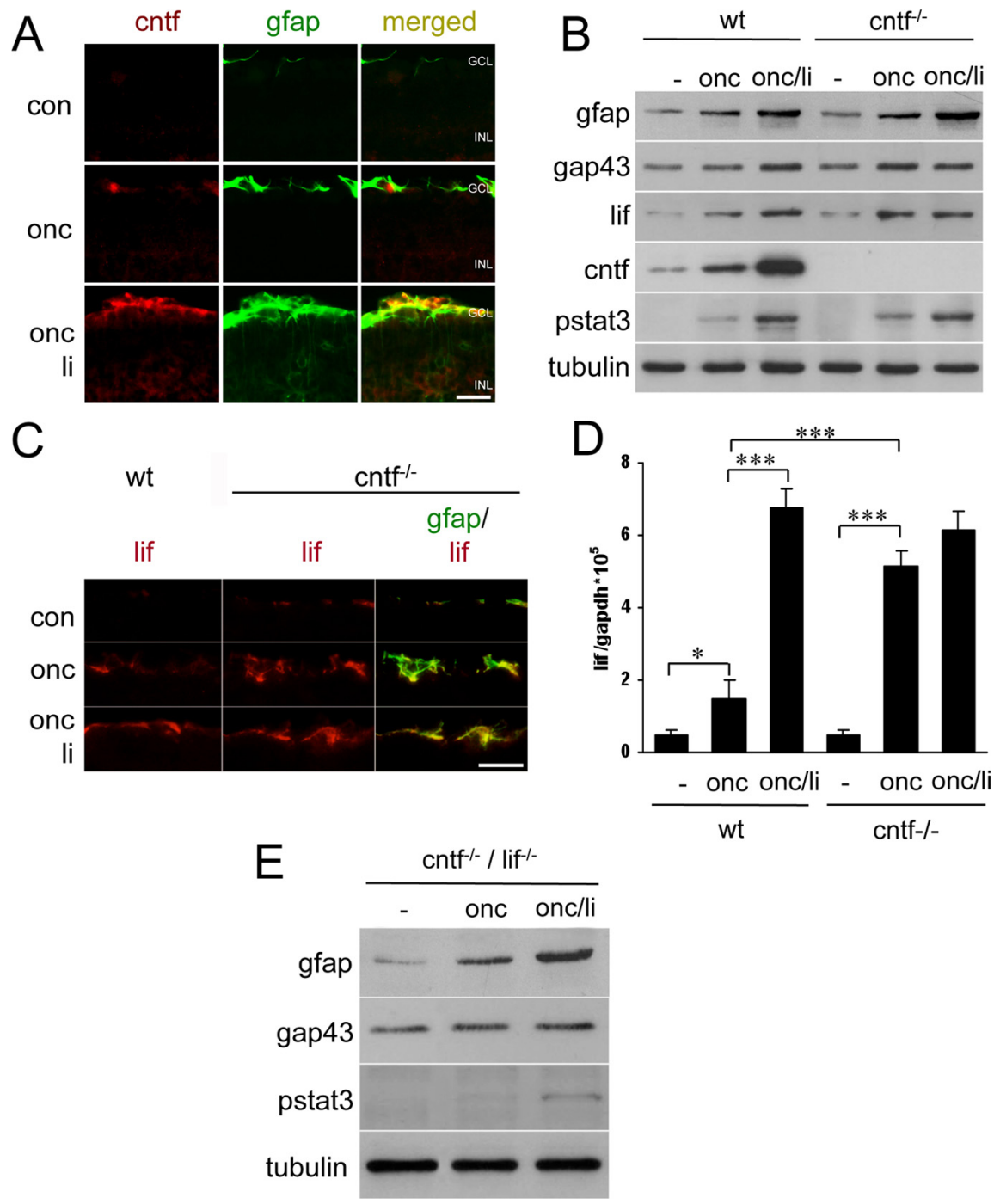

Figure 1. Changes in the expression of CNTF and LIF, as well as JAK/STAT3 pathway activation, in the murine retina after ONC and LI. A, Immunohistochemical staining of an untreated control retina (con), a retina $5 \mathrm{~d}$ after optic nerve crush (onc), and onc + cntf $^{-1-}$ ) mice $5 \mathrm{~d}$ after onc, onc + li, or no previous treatment (con) using specific antibodies against GFAP, GAP43, LIF, untreated control retina, a retina $5 \mathrm{~d}$ after onc, and after onc + li of wt and cntf ${ }^{-1-}$ mice using an anti-LIF (R\&D) (red) and anti-GFAP antibody (green). Scale bar, $25 \mu \mathrm{m}$. D, Quantitative real-time PCR. LIF expression levels were quantified relative to analysis of retinal lysates from CNTF/LIF double knock-out ( $\mathrm{cntf}^{-1-} /$ lif $^{-1-}$ ) mice $5 \mathrm{~d}$ after onc, onc $+\mathrm{li}$, or no previous treatment

\section{Results}

Regulation of CNTF and LIF expression and their contribution to the acquisition of a regenerative state of RGCs after ONC + LI

In rats LI or zymosan treatment strongly induces CNTF expression in retinal glial cells and we have recently demonstrated that CNTF is a potent neurite outgrowth-promoting factor for mature RGCs of both rats and mice when applied in vivo or in vitro (Müller et al., 2007, 2009). We, therefore, investigated whether $\mathrm{ONC}$ or $\mathrm{ONC}+\mathrm{LI}$ altered retinal CNTF expression in mice. As determined by Western blotting and immunochemistry, CNTF and GFAP expression was moderately upregulated $5 \mathrm{~d}$ after ONC and was further increased by an additional LI (Fig. $1 A, B$ ). Immu- 

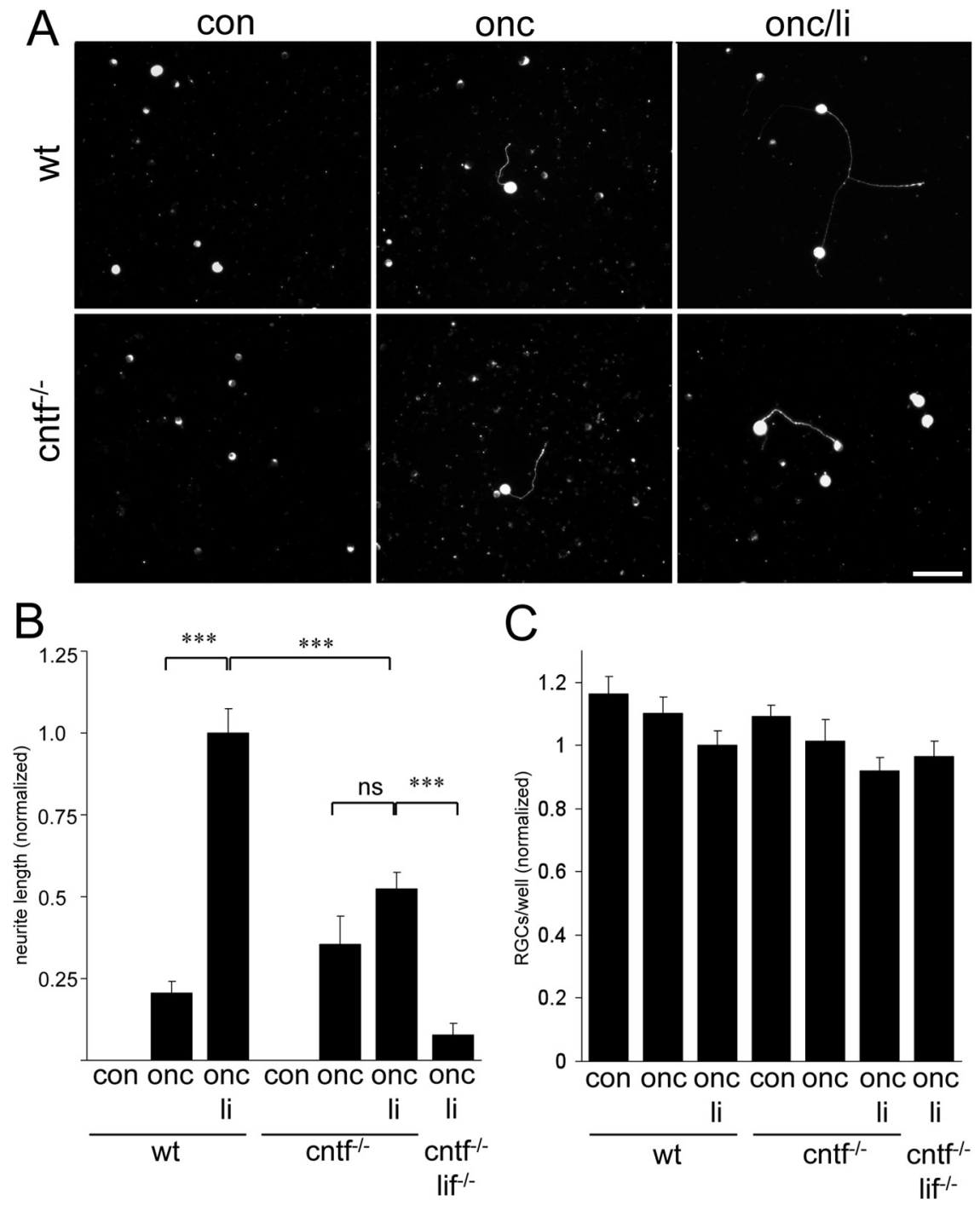
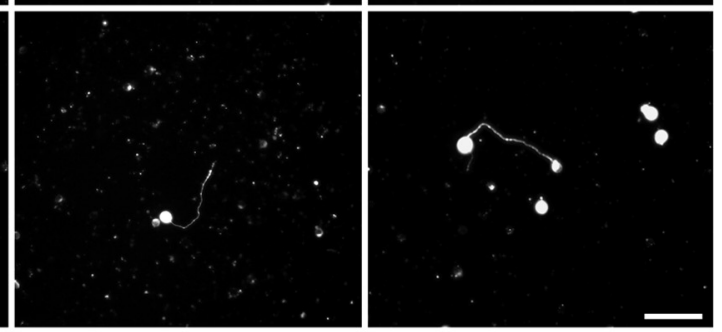

C

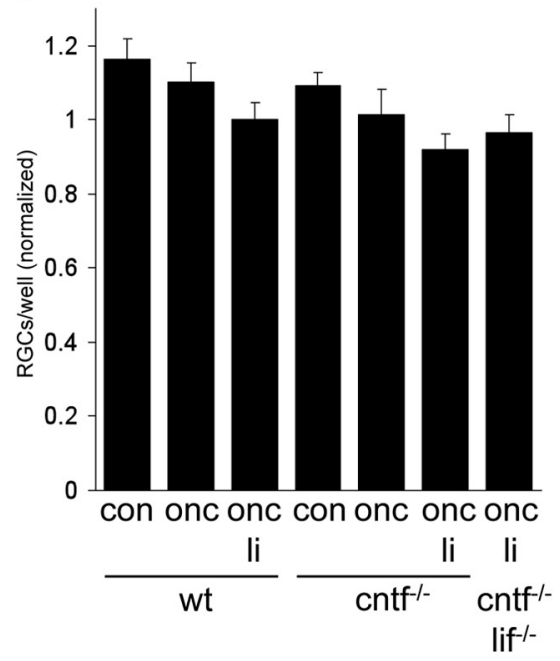

Figure 2. Regenerative state of RGCs 5 d after ONC and ONC + LI in mature wild-type and CNTF-deficient mice. $A$, Dissociated retinal cell cultures immunostained with an antibody against $\beta$ III-tubulin, showing spontaneously regenerating $\mathrm{RGCs}$ after $24 \mathrm{~h}$ in culture. Wild-type (wt) and CNTF-deficient ( $\mathrm{cntf}^{-/-}$) mice had received ONC (onc), 0NC + LI (onc + li), or no treatment (con) $5 \mathrm{~d}$ previously. Scale bar, $50 \mu \mathrm{m}$. B, Quantitation of neurite outgrowth of groups in $\boldsymbol{A}$ and of CNTF/LIF double knock-out (cntf ${ }^{-/-}$/ lif $^{-/-}$) mice $5 \mathrm{~d}$ after ONC $+\mathrm{LI}$, indicated as average neurite length per RGC. C, Quantitation of RGCs per well after $24 \mathrm{~h}$ in culture, demonstrating no significant differences between groups. Significance between groups: ${ }^{* * *} p<0.001$; ns., nonsignificant.

nohistochemical analysis located most CNTF expression to GFAP-positive retinal astrocytes (Fig. 1A). Consistent with our previous report in rats (Müller et al., 2007), CNTF was not detected in microglia or macrophages (supplemental Fig. 3, available at www.jneurosci.org as supplemental material). CNTF expression levels in the retina were correlated with an activation of CNTF's major downstream signaling pathway (JAK/STAT3) indicated by increased levels of pSTAT3 in the nuclei of RGCs (Fig. $1 B$; supplemental Fig. $1 A$, available at www.jneurosci.org as supplemental material). Less pSTAT3 staining than in RGCs was also detected in cells of the inner nuclear layer (supplemental Fig. $1 A$, available at www.jneurosci.org as supplemental material). Interestingly, quantitative real-time PCR, Western-blotting, and immunohistochemical analyses also showed a slight upregulation of retinal expression of LIF after ONC and a much stronger upregulation after additional LI (Fig. $1 B-D)$. As demonstrated by immunohistochemistry using two different antibodies against the cytokine, LIF expression colocalized in the retina with GFAP-positive astrocytes (Fig. $1 C$; supplemental Fig. $1 B$, available at www.jneurosci.org as supplemental material). Since macrophage-derived oncomodulin was proposed to be the principal mediator of LI effects (Yin et al., 2006), we also tested RNA expression levels of oncomodulin. Consistent with results previously published in rats (Hauk et al., 2008) oncomodulin levels were detected in retinal/vitreal samples but did not measurable change in the inner eye after ONC alone or ONC + LI compared with untreated controls in mice. In contrast, levels of CD68 were markedly increased after $\mathrm{ONC}+\mathrm{LI}$ in these samples (supplemental Fig. $1 C$ ), verifying the infiltration and activation of macrophages/microglia after LI treatment. The induction of CNTF and LIF expression was correlated with an upregulation of growth-associated protein 43 (GAP43) expression (Fig. 1 B), suggesting the transition of RGCs into an active regenerative state after ONC + LI. To functionally assess the regenerative state of RGCs we prepared retinal cell cultures $5 \mathrm{~d}$ after ONC, ONC+LI, or ONC + intravitreal injection of zymosan, which is typically before the onset of cell death after ONC. The average length of spontaneously outgrowing neurites of RGCs was quantified after $24 \mathrm{~h}$ in culture as previously described (Müller et al., 2007, 2009). The number of RGCs per well was not significantly different between groups (Fig. $2 C$; supplemental Fig. $2 B$, available at www.jneurosci.org as supplemental material). Untreated animals showed almost no spontaneous neurite outgrowth within $24 \mathrm{~h}$ of culture and RGCs from animals that were subjected to ONC $5 \mathrm{~d}$ before regenerated few, mostly short neurites (Fig. $2 A, B)$. In contrast, axotomized RGCs of animals that were subjected to ONC $+\mathrm{LI}$ or $\mathrm{ONC}+$ zymosan generated, on average, neurites that were significant longer than those in animals that underwent ONC only (Fig. $2 A$, B; supplemental Fig. $2 A$, available at www.jneurosci.org as supplemental material), verifying that LI or intravitreal application of zymosan markedly stimulate neurite growth of murine RGCs as early as $5 \mathrm{~d}$ after surgery.

To evaluate the contribution of CNTF to the LI-mediated effects, we performed the same experiments as described above for wild-type animals, in parallel with CNTF-deficient mice. The lack of CNTF expression in these mice was verified by Western blot analysis (Fig. $1 B$ ), by RT-PCR (data not shown), and immunohistochemistry (supplemental Fig. 3, available at www. jneurosci.org as supplemental material). Surprisingly, compared with wild-type mice, retinas of CNTF-deficient mice displayed a stronger upregulation of LIF expression $5 \mathrm{~d}$ after ONC (Fig. $1 \mathrm{~B}-$ $D)$. However, contrary to wild-type animals, an additional LI increased expression levels of LIF only slightly further in CNTFdeficient mice, thus resulting in similar expression levels of LIF after ONC alone and ONC + LI (Fig. $1 B-D$ ). In CNTF-deficient 
mice the expression levels of GAP43 and pSTAT3 were correlated with the expression of LIF, suggesting that LIF expression may have induced most of the JAK/ STAT3 activation and partially compensated for the CNTF deficiency. The regenerative state of RGCs from CNTFdeficient mice was functionally determined as described above in retinal cultures. Neurite outgrowth was only $\sim 50 \%$ of that observed in wild-type mice, indicating that CNTF plays an important active role in mediating the LI-induced promotion of neurite outgrowth (Fig. $2 A, B)$. Consistent with LIF expression, neurite outgrowth was significantly more robust in CNTF-deficient mice after ONC compared with identically treated wildtype animals and was only slightly, but nonsignificantly lower than neurite outgrowth measured after an additional LI (Fig. 2A, B). The number of RGCs in these cultures was similar in all tested groups (Fig. 2C), demonstrating that the deficiency of CNTF did not affect the survival of RGCs $5 \mathrm{~d}$ after optic nerve injury and $1 \mathrm{~d}$ in culture.

LI effects on axon regeneration in the optic nerve and neuroprotection are strongly compromised in CNTF-deficient and absent in CNTF/LIF double knock-out mice

To measure the contribution of CNTF to the neuroprotective and axon growthpromoting effects of LI on axotomized RGCs in vivo, the number of regenerating axons and surviving RGCs in wild-type and CNTF-deficient mice after ONC and ONC + LI were quantified and compared with each other (Fig. 3B,C). Wild-type animals that were subjected to ONC only showed little axonal regeneration beyond the lesion site, whereas LI-treated mice displayed the strongest regeneration. In these animals the longest axons averaged a length of $1.9 \pm 0.15 \mathrm{~mm}$ beyond the lesion by $14 \mathrm{~d}$ after surgery. In contrast, in CNTF-deficient mice only approximately half as many axons regenerated into the distal part of the injured optic nerve after LI (Fig. $3 A, B$ ) compared with wild-type mice. The longest axons reached on average a length of only $0.89 \pm 0.09 \mathrm{~mm}$ by $14 \mathrm{~d}$ after ONC + LI. In these mice, LI was also much less effective at protecting axotomized RGCs from cell death compared with wild-type animals (Fig. 3C). Consistent with results shown in Figure 2, CNTF-deficient mice displayed a moderate regeneration into the optic nerve after ONC, which was greater than identically treated wild-type mice. Since in these
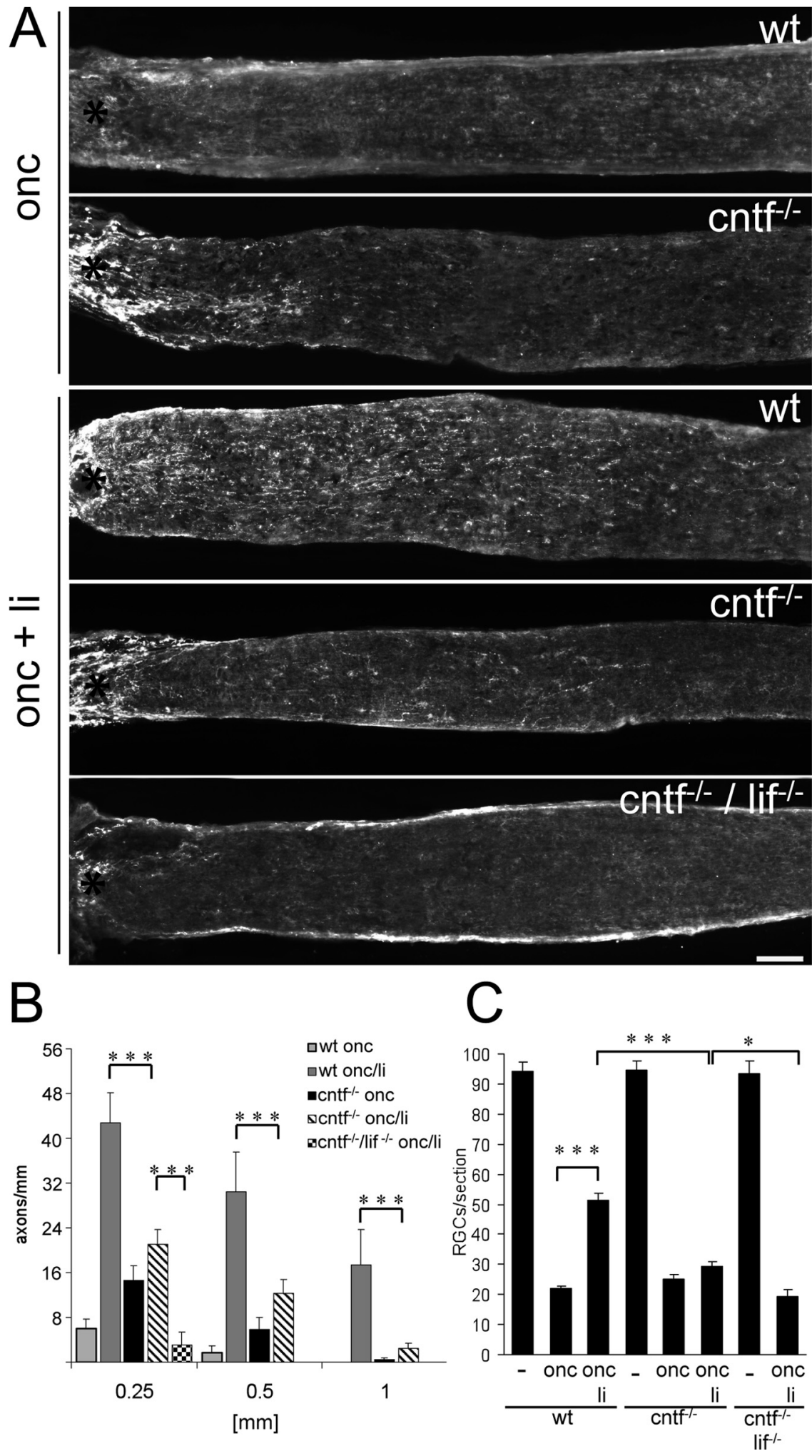

Figure 3. Axon regeneration and survival of RGCs in vivo after Ll in wild-type, CNTF-deficient and CNTF/LIF double knock-out mice. $\boldsymbol{A}$, Longitudinal sections through the optic nerve showing GAP43-positive axons distal to the injury site (asterisk) in

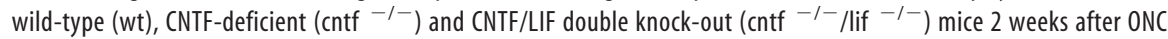
alone (onc) or ONC $+\mathrm{LI}$ (onc + li). Scale bar, $100 \mu \mathrm{m}$. B, Quantification of axon regeneration (number of axons growing $0.25,0.5$, and $1 \mathrm{~mm}$ beyond the injury site per optic nerve) 2 weeks after ONC alone or ONC + LI. C, Quantification of surviving RGCs ( $\beta$ III-tubulin-positive RGCs per retinal cross-section) 2 weeks after ONC alone, ONC $+\mathrm{LI}$, or untreated retinas $(-) .{ }^{*} p<0.05 ;{ }^{* * *} p<0.001$. 
cases (without LI) axon regeneration was correlated with LIF expression (Fig. 1) we speculated that LIF may function as an additional contributing factor partially compensating for the deficiency in CNTF. To test this hypothesis, we performed ONC + LI in CNTF/LIF double knock-out mice. Although LI exerted also in CNTF/LIF-deficient mice a similar robust activation of macrophages/microglia as observed in wild-type or CNTF-deficient mice (supplemental Fig. 3, available at www.jneurosci.org as supplemental material) and was also associated with an activation of retinal glia indicated by an upregulation of retinal GFAP expression (Fig. $1 E$ ), no neuroprotective effects (Fig. $3 C$ ) or improved axon regeneration were observed in CNTF/LIF-deficient mice $14 \mathrm{~d}$ after LI (Fig. $3 A, B$ ). In fact, the number and length of regenerating axons in the crushed optic nerve after LI appeared to be even somewhat lower than in wild-type animals subjected to ONC only (Fig. 3B). Consistent with these results, no upregulation in retinal GAP43 expression was seen after ONC alone or ONC + LI compared with untreated controls in the double knock-out mice (Fig. $1 E$ ). Furthermore, retinal levels of pSTAT3 remained very low and were barely elevated after ONC + LI treatment (Fig. 1E). RGCs of CNTF/LIF double knock-out mice showed also very little spontaneous neurite outgrowth when cultured $5 \mathrm{~d}$ after ONC + LI (Fig. $2 B$ ) or ONC + intravitreal application of zymosan (supplemental Fig. $2 A$, available at www. jneurosci.org as supplemental material), whereas the survival of RGCs in these cultures was not significantly affected (Fig. 2C; supplemental Fig. $2 B$, available at www.jneurosci.org as supplemental material).

\section{LIF is a potent neurite growth-promoting factor}

It was recently shown that CNTF potently stimulates neurite outgrowth of mature RGCs in culture with maximal effects at 200 $\mathrm{ng} / \mathrm{ml}$ (Müller et al., 2009). To test whether LIF exerts effects of similar potency as CNTF we prepared dissociated retinal cell cultures and exposed RGCs to increasing concentrations of LIF. LIF markedly increased neurite outgrowth of RGCs in a concentration-dependent manner (Fig. 4A). Effects of LIF were maximal and similar potent as CNTF at concentrations $\geq 200 \mathrm{ng} / \mathrm{ml}$ and were abrogated in the presence of an anti-LIF antibody that was added to the culture medium (Fig. $4 A$ ). The increase in neurite outgrowth was not a consequence of neuroprotective influences, since the numbers of RGCs per well did not significantly differ between any of the groups tested after $3 \mathrm{~d}$ in culture (Fig. $4 \mathrm{~B}$ ).

\section{Discussion}

After optic nerve crush, adult rat RGC axons normally fail to regenerate beyond the lesion site. However, when optic nerve crush is combined with either intravitreal injections of $\beta / \gamma$ crystallins, zymosan, Pam3Cys or LI, cell death of axotomized RGCs is markedly delayed and axons grow into the distal part of the injured optic nerve (Fischer et al., 2000, 2001; Leon et al., 2000; Lorber et al., 2002; Pernet and Di Polo, 2006; Hauk et al., 2009). We have recently shown that glial-derived CNTF is one of the major mediators of this phenomenon (Müller et al., 2007, 2009; Fischer, 2008). However, a role for CNTF or its downstream signaling pathway (JAK/STAT3 pathway) in mediating these effects has been questioned by others (Yin et al., 2003, 2006; Cui et al., 2008, 2009). Among the arguments raised against a significant role of CNTF were the reports that a single intravitreal injection of an anti-CNTF antibody did not compromise LIinduced regeneration into the optic nerve (Leon et al., 2000; Cui et al., 2008). However, these data may be explained by the short half-life time of active antibodies in the vitreous body used in
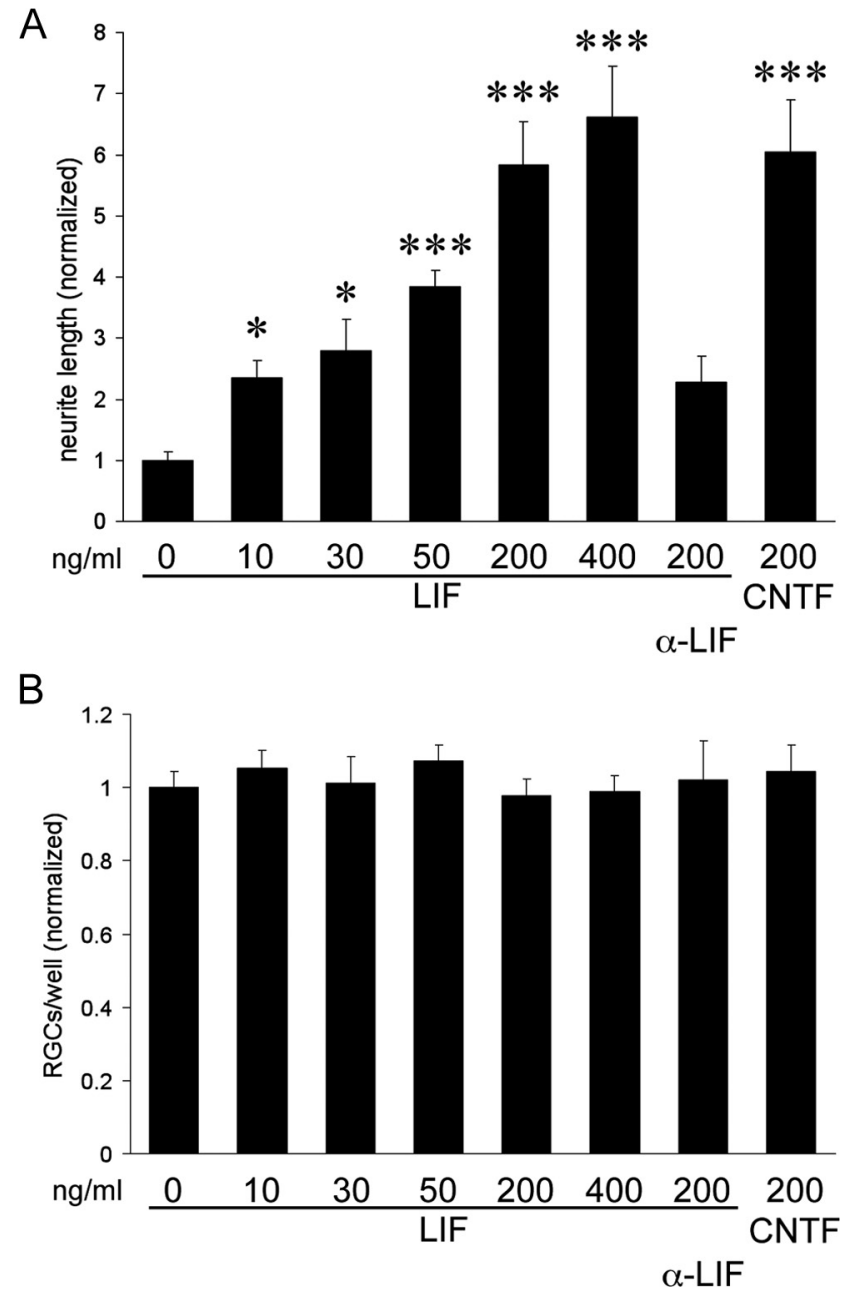

Figure 4. Neurite growth-promoting effects of LIF and CNTF on mature RGCs in culture. $A$, Quantitation of neurite outgrowth of RGCs in the presence of increasing concentrations of LIF (as indicated), CNTF and an anti-LIF-antibody ( $\alpha$-LIF) for $3 \mathrm{~d}$. Values were normalized to untreated controls. Treatment effects compared with the group treated with vehicle only. ${ }^{*} p<0.05 ;{ }^{* * *} p<0.001$. B, Quantitation of RGCs per well after $3 \mathrm{~d}$ in culture, demonstrating no significant differences between groups.

these studies, whereas CNTF is continuously released from retinal glia (Fischer, 2008).

To shed further light on this ongoing controversy, and to circumvent limitations caused by the restricted half-life times and potential indirect effects of intravitreally applied compounds or peptides we tested the contribution of CNTF by taking advantage of the availability of CNTF knock-out mice and the fact that LI exerts strong neuroprotective and axon growth-promoting effects on axotomized RGCs in mice as well (Lorber et al., 2002, 2005b). We showed here, consistent with results previously obtained in rats that, (1) CNTF expression is markedly upregulated in retinal astrocytes in mice, (2) CNTF upregulation was paralleled by increased levels of pSTAT3 in RGCs, and (3) CNTF expression and retinal pSTAT3 levels were correlated with the regenerative state of RGCs after ONC + LI. These data suggest that the molecular mechanisms underlying LI effects in mice and in rats are similar. The current study supports also the hypothesis that CNTF is a key factor for mediating the beneficial effects of LI, since CNTF deficiency strongly compromised LI-stimulated neurite outgrowth in vitro $5 \mathrm{~d}$ after $\mathrm{ONC}$, as well as neuroprotection and axon regeneration into the optic nerve in vivo $14 \mathrm{~d}$ after 
surgery. However, CNTF does not appear to be the only contributing factor, as we measured also a robust upregulation of LIF expression in retinal astrocytes. LIF expression in astrocytes has been also shown after cortical brain injury or focal cerebral ischemia (Banner et al., 1997; Suzuki et al., 2000). LIF possesses a signal sequence and is released by cells (Knight, 2001). Since all components of a fully functional LIF receptor are expressed in RGCs (Sarup et al., 2004) and since LIF, like CNTF, strongly stimulates neurite outgrowth of RGCs in culture as shown in the current study, it is conceivable that glial-derived LIF contributes to the LI-induced stimulation of axon regeneration in the optic nerve. This hypothesis is supported by our observation that ONC in CNTF-deficient mice induced a stronger LIF expression compared with wild-type animals which was paralleled by increased neurite outgrowth in vitro and axon regeneration after ONC in vivo. The reason for the stronger upregulation of LIF in CNTFdeficient mice compared with wild-type animals after ONC remains to be unraveled, but it is consistent with a compensatory upregulation of gp130-signaling in CNTF-deficient mice that has also been observed in the denervated superior colliculus following optic nerve damage (Martin et al., 2003). The complete absence of any beneficial LI effects in CNTF/LIF-deficient mice, which was associated with an almost complete loss of retinal STAT3 activation, suggests that CNTF- and LIF-induced gp130 signaling is essential for the neuroprotective and axon regenerative effects of intraocular inflammatory stimulation. Moreover, the facts that LI-induced infiltration of macrophages into the eye and activation of microglia and astrocytes/Müller cells were not reduced in CNTF/LIF double knock-out mice, but that the beneficial effects of LI were absent in these animals, argue for a direct effect of both cytokines on RGCs. These data also suggest that macrophage activation alone is insufficent to stimulate axon regeneration. A direct effect of the cytokines is further supported by the activation of CNTF's and LIF's major downstream signaling pathway (JAK/STAT3 pathway) in RGCs and the strong neurite outgrowth-promoting effects of CNTF and LIF on mature RGCs in culture shown in the current study and previously (Müller et al., 2009). Despite the essential contribution of CNTF and LIF in mediating the beneficial effects of inflammatory stimulation in the eye, the data presented in the current study do not exclude the contribution of additional factors, which may synergistically enhance the beneficial effects of CNTF and LIF, such as astrocytederived ApoE (Lorber et al., 2009) or other factors. However, oncomodulin expression levels were detected, but not measurably upregulated in the inner eye of mice after optic nerve injury and LI. These data are consistent with previous findings in rats (Hauk et al., 2008; Park et al., 2009) and therefore also argue against a claimed principal role of oncomodulin in mediating the beneficial effects of inflammatory stimulation (Yin et al., 2006; Benowitz and Yin, 2007, 2008; Cui et al., 2009). Furthermore, it should be noted that (1) intravitreal application of antioncomodulin antibodies did not compromise LI effects, (2) oncomodulin alone is insufficient to enhance axon regeneration of mature RGCs in vitro and in vivo and instead requires additional cAMP elevation, and (3) the effects of oncomodulin + cAMP elevation are markedly weaker than those of CNTF alone (Müller et al., 2007, 2009; Fischer, 2008).

In conclusion, the results of the present study support the hypothesis that astrocytes are the major source of factors mediating the neuroprotective and axon regeneration-promoting effects of inflammatory stimulation in the eye. Furthermore, they provide additional confirmatory evidence for CNTF being a direct key mediator of these beneficial effects and identify LIF as another significant contributing factor.

\section{References}

Banner LR, Moayeri NN, Patterson PH (1997) Leukemia inhibitory factor is expressed in astrocytes following cortical brain injury. Exp Neurol 147:1-9.

Benowitz L, Yin Y (2008) Rewiring the injured CNS: lessons from the optic nerve. Exp Neurol 209:389-398.

Benowitz LI, Yin Y (2007) Combinatorial treatments for promoting axon regeneration in the CNS: strategies for overcoming inhibitory signals and activating neurons' intrinsic growth state. Dev Neurobiol 67:1148-1165.

Berkelaar M, Clarke DB, Wang YC, Bray GM, Aguayo AJ (1994) Axotomy results in delayed death and apoptosis of retinal ganglion cells in adult rats. J Neurosci 14:4368-4374.

Berry M, Carlile J, Hunter A (1996) Peripheral nerve explants grafted into the vitreous body of the eye promote the regeneration of retinal ganglion cell axons severed in the optic nerve. J Neurocytol 25:147-170.

Berry M, Carlile J, Hunter A, Tsang W, Rosenstiel P, Sievers J (1999) Optic nerve regeneration after intravitreal peripheral nerve implants: trajectories of axons regrowing through the optic chiasm into the optic tracts. J Neurocytol 28:721-741.

Cui Q, Benowitz L, Yin Y (2008) Does CNTF mediate the effect of intraocular inflammation on optic nerve regeneration? Brain 131:e96; author reply e97.

Cui Q, Yin Y, Benowitz LI (2009) The role of macrophages in optic nerve regeneration. Neuroscience 158:1039-1048.

Fischer D (2008) CNTF, a key factor mediating the beneficial effects of inflammatory reactions in the eye. Brain 131:e97.

Fischer D, Pavlidis M, Thanos S (2000) Cataractogenic lens injury prevents traumatic ganglion cell death and promotes axonal regeneration both in vivo and in culture. Invest Ophthalmol Vis Sci 41:3943-3954.

Fischer D, Heiduschka P, Thanos S (2001) Lens-injury-stimulated axonal regeneration throughout the optic pathway of adult rats. Exp Neurol 172:257-272.

Fischer D, He Z, Benowitz LI (2004a) Counteracting the Nogo receptor enhances optic nerve regeneration if retinal ganglion cells are in an active growth state. J Neurosci 24:1646-1651.

Fischer D, Petkova V, Thanos S, Benowitz LI (2004b) Switching mature retinal ganglion cells to a robust growth state in vivo: gene expression and synergy with RhoA inactivation. J Neurosci 24:8726-8740.

Fischer D, Hauk TG, Müller A, Thanos S (2008) Crystallins of the beta/ gamma-superfamily mimic the effects of lens injury and promote axon regeneration. Mol Cell Neurosci 37:471-479.

Hauk TG, Müller A, Lee J, Schwendener R, Fischer D (2008) Neuroprotective and axon growth promoting effects of intraocular inflammation do not depend on oncomodulin or the presence of large numbers of activated macrophages. Exp Neurol 209:469-482.

Hauk TG, Leibinger M, Müller A, Andreadaki N, Knippschild U, Fischer D (2009) Intravitreal application of the Toll-like receptor 2 agonist Pam3Cys stimulates axon regeneration in the mature optic nerve. Invest Ophthalmol Vis Sci. Advance online publication. Retrieved Oct. 28, 2009. doi:10.1167/iovs.09-4203.

Heinrich PC, Behrmann I, Haan S, Hermanns HM, Müller-Newen G, Schaper F (2003) Principles of interleukin (IL)-6-type cytokine signalling and its regulation. Biochem J 374:1-20.

Holtmann B, Wiese S, Samsam M, Grohmann K, Pennica D, Martini R, Sendtner M (2005) Triple knock-out of CNTF, LIF, and CT-1 defines cooperative and distinct roles of these neurotrophic factors for motoneuron maintenance and function. J Neurosci 25:1778-1787.

Knight D (2001) Leukaemia inhibitory factor (LIF): a cytokine of emerging importance in chronic airway inflammation. Pulm Pharmacol Ther 14:169-176.

Leon S, Yin Y, Nguyen J, Irwin N, Benowitz LI (2000) Lens injury stimulates axon regeneration in the mature rat optic nerve. J Neurosci 20:4615-4626.

Lorber B, Berry M, Logan A, Tonge D (2002) Effect of lens lesion on neurite outgrowth of retinal ganglion cells in vitro. Mol Cell Neurosci 21:301-311.

Lorber B, Berry M, Logan A (2005a) Lens injury stimulates adult mouse retinal ganglion cell axon regeneration via both macrophage- and lensderived factors. Eur J Neurosci 21:2029-2034. 
Lorber B, Hendriks WJ, Van der Zee CE, Berry M, Logan A (2005b) Effects of LAR and PTP-BL phosphatase deficiency on adult mouse retinal cells activated by lens injury. Eur J Neurosci 21:2375-2383.

Lorber B, Berry M, Logan A (2008) Different factors promote axonal regeneration of adult rat retinal ganglion cells after lens injury and intravitreal peripheral nerve grafting. J Neurosci Res 86:894-903.

Lorber B, Berry M, Douglas MR, Nakazawa T, Logan A (2009) Activated retinal glia promote neurite outgrowth of retinal ganglion cells via apolipoprotein E. J Neurosci Res 87:2645-2652.

Martin A, Hofmann HD, Kirsch M (2003) Glial reactivity in ciliary neurotrophic factor-deficient mice after optic nerve lesion. J Neurosci 23:5416-5424.

Müller A, Hauk TG, Fischer D (2007) Astrocyte-derived CNTF switches mature RGCs to a regenerative state following inflammatory stimulation. Brain 130:3308-3320.

Müller A, Hauk TG, Leibinger M, Marienfeld R, Fischer D (2009) Exogenous CNTF stimulates axon regeneration of retinal ganglion cells partially via endogenous CNTF. Mol Cell Neurosci 41:233-246.

Park KK, Hu Y, Muhling J, Pollett MA, Dallimore EJ, Turnley AM, Cui Q, Harvey AR (2009) Cytokine-induced SOCS expression is inhibited by cAMP analogue: impact on regeneration in injured retina. Mol Cell Neurosci 41:313-324.

Pernet V, Di Polo A (2006) Synergistic action of brain-derived neurotrophic factor and lens injury promotes retinal ganglion cell survival, but leads to optic nerve dystrophy in vivo. Brain 129:1014-1026.

Sarup V, Patil K, Sharma SC (2004) Ciliary neurotrophic factor and its receptors are differentially expressed in the optic nerve transected adult rat retina. Brain Res 1013:152-158.

Sendtner M, Götz R, Holtmann B, Escary JL, Masu Y, Carroll P, Wolf E, Brem G, Brület P, Thoenen H (1996) Cryptic physiological trophic support of motoneurons by LIF revealed by double gene targeting of CNTF and LIF. Curr Biol 6:686-694.

Suzuki S, Tanaka K, Nogawa S, Ito D, Dembo T, Kosakai A, Fukuuchi Y (2000) Immunohistochemical detection of leukemia inhibitory factor after focal cerebral ischemia in rats. J Cereb Blood Flow Metab 20:661-668.

Taupin JL, Pitard V, Dechanet J, Miossec V, Gualde N, Moreau JF (1998) Leukemia inhibitory factor: part of a large ingathering family. Int Rev Immunol 16:397-426.

Yin Y, Cui Q, Li Y, Irwin N, Fischer D, Harvey AR, Benowitz LI (2003) Macrophage-derived factors stimulate optic nerve regeneration. J Neurosci 23:2284-2293.

Yin Y, Henzl MT, Lorber B, Nakazawa T, Thomas TT, Jiang F, Langer R, Benowitz LI (2006) Oncomodulin is a macrophage-derived signal for axon regeneration in retinal ganglion cells. Nat Neurosci 9:843852. 